\title{
Tracoma em crianças do ensino fundamental no município de Bauru - Estado de São Paulo, Brasil
}

\author{
Prevalence of trachoma among school children in Bauru - São Paulo State, Brazil \\ Lucieni Cristina Barbarini Ferraz ${ }^{1}$, Silvana Artioli Schellin ${ }^{1}$, Carlos Roberto Padovan² ${ }^{2}$, Norma Helen Medina ${ }^{3}$, Ivete Dalben ${ }^{4}$
}

\section{RESUMO}

Objetivo: Foi realizado um estudo transversal, por amostragem de escolares, frequentadores das escolas estaduais de Bauru, São Paulo, com o objetivo de detectar possíveis portadores de tracoma.

Métodos: Foram examinadas 1.749 crianças do ensino fundamental, de 6 a 14 anos, durante o ano de 2005. As escolas foram escolhidas por sorteio, de acordo com o número de estudantes e com a localização da escola dentro do setor da cidade (centro, região intermediária e periferia). O diagnóstico dos casos foi clínico, seguindo as normas da Organização Mundial de Saúde (OMS) confirmado laboratorialmente pelo método da imunofluorescência direta para detecção de Chlamydia trachomatis.

Resultados: A prevalência de tracoma inflamatório foi de 3,8\%, tendo sido diagnosticado tracoma folicular em 3,7\% e tracoma intenso em 0,06\% das crianças examinadas. As crianças com tracoma moravam principalmente na região intermediária e periférica da cidade, áreas onde o fornecimento de água pode ser descontínuo e o tratamento do esgoto pode estar ausente. Um programa de controle foi desenvolvido e os comunicantes foram diagnosticados e tratados.

Conclusão: A prevalência de tracoma inflamatório em Bauru - Estado de São Paulo - é de 3,8\%. Além da oportunidade de reconhecer e tratar os portadores e contatantes, os autores realçam o fato positivo da mobilização da comunidade bauruense no sentido de prevenir a cegueira pelo tracoma.

Descritores: Tracoma/epidemiologia; Tracoma/diagnóstico; Cegueira/pre venção \& controle:Saúdeocular:Imunofluorescência:Azitromicina/usoterapêutico; Tracoma/quimioterapia; Fatores socioeconômicos; Pré-escolar; Criança

\section{ABSTRACT}

Purpose: A survey was carried out with schoolchildren in Bauru, São Paulo State. The purpose of this study was to determine the prevalence of trachoma. Methods: One thousand, seven hundred and forty-nine children from 6 to 14 years old were examined during the year of 2005. The schools were randomly chosen, according to the number of schoolchildren and the school region according to the local of the city (downtown, middle and periphery). The diagnosis was performed according to the World Health Organization (WHO) protocol and patients with clinical trachoma detection were submitted to laboratory confirmation by direct smear fluorescent antibodies cytology for Chlamydia trachomatis.

Results: Trachoma prevalence was 3.8\%. Follicular trachoma was diagnosed in $3.7 \%$ of children, and intense trachoma in one child (0.06\%). Children with trachoma were living mainly in the middle and peripheral region of the city, places with discontinuous water supply and absence of sewerage system in part of the residences.

Conclusion: Trachoma prevalence in Bauru - São Paulo State - is 3.8\%. The authors point out the survey results and also treated trachoma carriers. Other positive aspect of the survey was the community mobilization to prevent trachoma blindness.

Keywords: Trachoma/epidemiology; Trachoma/diagnosis; Blindness/prevention \& control. Eye health. Fluorescent antibody technique: Azithromycin/therapeutic use; Trachoma/drug therapy; Socioeconomicfactors; Child, preschool; Child

\section{INTRODUÇÃO}

0 acoma é uma ceratoconjuntivite crônica, causada pela bactéria Chlamydia trachomatis. A doença é contagiosa e, potencialmente, causa cegueira ${ }^{(1-2)}$.

O tracoma foi considerado "erradicado" do Estado de São Paulo desde a década de 70. Entretanto, a partir de 1982 novos casos começaram a ser diagnosticados no município de Bebedouro, região noroeste do estado(3)

Trabalho realizado na Faculdade de Medicina de Botucatu, Botucatu (SP) - Brasil. ${ }^{1}$ Médico, Faculdade de Medicina de Botucatu, Universidade Estadual Paulista - UNESP, Bauru (SP), Brasil.

. Estatística, Instituto de Biociências, Universidade Estaduat Paulista UNESP, Bauru (SP), Brasil.

3 Médico, Centro de Vigilância Enidemiológica, Secretaria de Estado da Saúde de São Paulo, São Paulo (SP) Brasil.

Sao Paulo (SP), Brasil. de Estadual Paulista - UNESP, Botucatu (SP), Brasil.

Endereço para correspondência: Silvana Artioli Schellini. DEP. OFT/ORL/CCP - Faculdade de Medicina de Botucatu - UNESP - Distrito de Rubião Jr, s/n - Botucatu (SP)

CEP 18618-970 - E-mail: sartioli@fmb.unesp.br

Recebido para publicação em 05.02.2010

Última versão recebida em 18.10.2010

Ultima versão recebida em 18.1
A cidade de Bauru está localizada na região centro-oeste do Estado de São Paulo, sendo um importante polo de desenvolvimento para a região. O primeiro dado oficial sobre tracoma em Bauru data de 1936, quando houve um inquérito em escolas primárias, entre crianças de 7 a 13 anos, em municípios do norte e oeste do Estado. A prevalência em escolares de Bauru era de $6,5 \%$, enquanto outras cidades ao redor registravam: 10,0\% em Botucatu, 43,5\% em Jaú, 26,1\% em Barra Bonita e 31,3\% em Ribeirão Preto; as taxas mais altas aconteceram justamente onde a produção cafeeira foi mais proeminente ${ }^{(4-5)}$.

Entretanto, desde ano 2000 não houve nenhum caso notificado na cidade de Bauru, havendo registros de casos esporádicos na região, principalmente em cidades como Cafelândia e Pirajuí, que fazem parte da Regional de Saúde de Bauru.

Desta forma, foi realizado este estudo que teve por objetivo conhecer se a doença está presente e a prevalência de tracoma em escolares na cidade de Bauru, São Paulo.

\section{MÉTODOS}

O presente estudo foi analisado pelo Comitê de Ética em Pesquisa com Seres Humanos da Faculdade de Medicina de Botucatu, tendo sido aprovado para execução, sendo também 
autorizado pela Diretoria Regional de Ensino de Bauru. A participação dos alunos foi feito mediante assinatura do Termo de consentimento livre e esclarecido pelos pais ou responsáveis legais.

Este foi um estudo transversal, utilizando amostra estratificada e aleatória de escolares que frequentavam as escolas estaduais do Município de Bauru (São Paulo), pesquisando-se a presença do tracoma.

O tamanho da amostra foi determinado pela estimativa da população de alunos para o ano de 2005, fornecida pela Diretoria Regional de Ensino, pela qual o total naquele ano seria de 14.057 alunos.

No município de Bauru existiam 51 escolas estaduais, das quais, 30 possuiam ensino de primeira à quarta série. As escolas investigadas foram escolhidas por sorteio, que levou em conta o número de escolas, o número de classes e o número de alunos da região de estudo, além de considerar os setores da cidade (centro, intermediário e periferia), conforme apresentado na figura 1. Assim, foram sorteadas 12 escolas, 3 no centro, 4 na região intermediária e 5 na periferia.

Nos meses de abril a maio, foram examinadas 1.749 crianças com idade entre 6 a 14 anos, que frequentam o ensino fundamental, de primeira à quarta séries.

Considerou-se caso de tracoma a criança portadora do diagnóstico clínico da doença.

O exame clínico foi realizado com uma lupa de 2,5 vezes de magnificação, sob iluminação com lanterna, avaliando-se alterações das pálpebras, cílios, conjuntiva tarsal e bulbar e córnea. As pálpebras superiores foram evertidas e a conjuntiva tarsal superior cuidadosamente examinada. O tracoma foi diagnosticado e classificado de acordo com os critérios da OMS para detecção epidemiológica da doença, apresentados no quadro 1.
O exame laboratorial para comprovação do agente etiológico foi feito usando-se a imunofluorescência direta com anticorpos monoclonais (IFD), tendo sido realizado em 13 amostras, escolhidas aleatoriamente, de crianças com diagnóstico clínico de tracoma inflamatório.

Foi elaborado junto ao Geoprocessamento do Departamento de Água e Esgoto (DAE) da cidade de Bauru um mapa das escolas estudadas, com a intenção de avaliar distribuição da moradia dos casos de escolares detectados como portadores de tracoma.

Os comunicantes foram examinados, tratados e acompanhados, seguindo normas da Organização Mundial da Saúde (OMS).

Os dados foram armazenados em planilha Excel. O processamento das análises estatísticas foi realizado utilizando o pacote estatístico SAEG-4.0 (Sistema de Análise Estatística e Genética) desenvolvido pela Universidade Federal de Viçosa, Minas Gerais. O estudo da associação entre tracoma e variáveis relativas foi realizado utilizando o teste de Goodman para contrastes entre e dentro de populações multinominais ${ }^{(6-7)}$.

Todas as conclusões, no presente trabalho, foram discutidas no nível de $5 \%$ de significância.

\section{RESULTADOS}

A amostra pretendida para a pesquisa foi calculada em 1.054 crianças. Entretanto, o total de crianças examinadas foi de 1.749 , pois para atingir o número desejado, muitas vezes havia necessidade de examinar uma nova classe e desta sala todas as crianças eram examinadas. A amostra aumentou para não rejeitarmos nenhuma criança, não poderíamos causar frustração em pais e alunos.

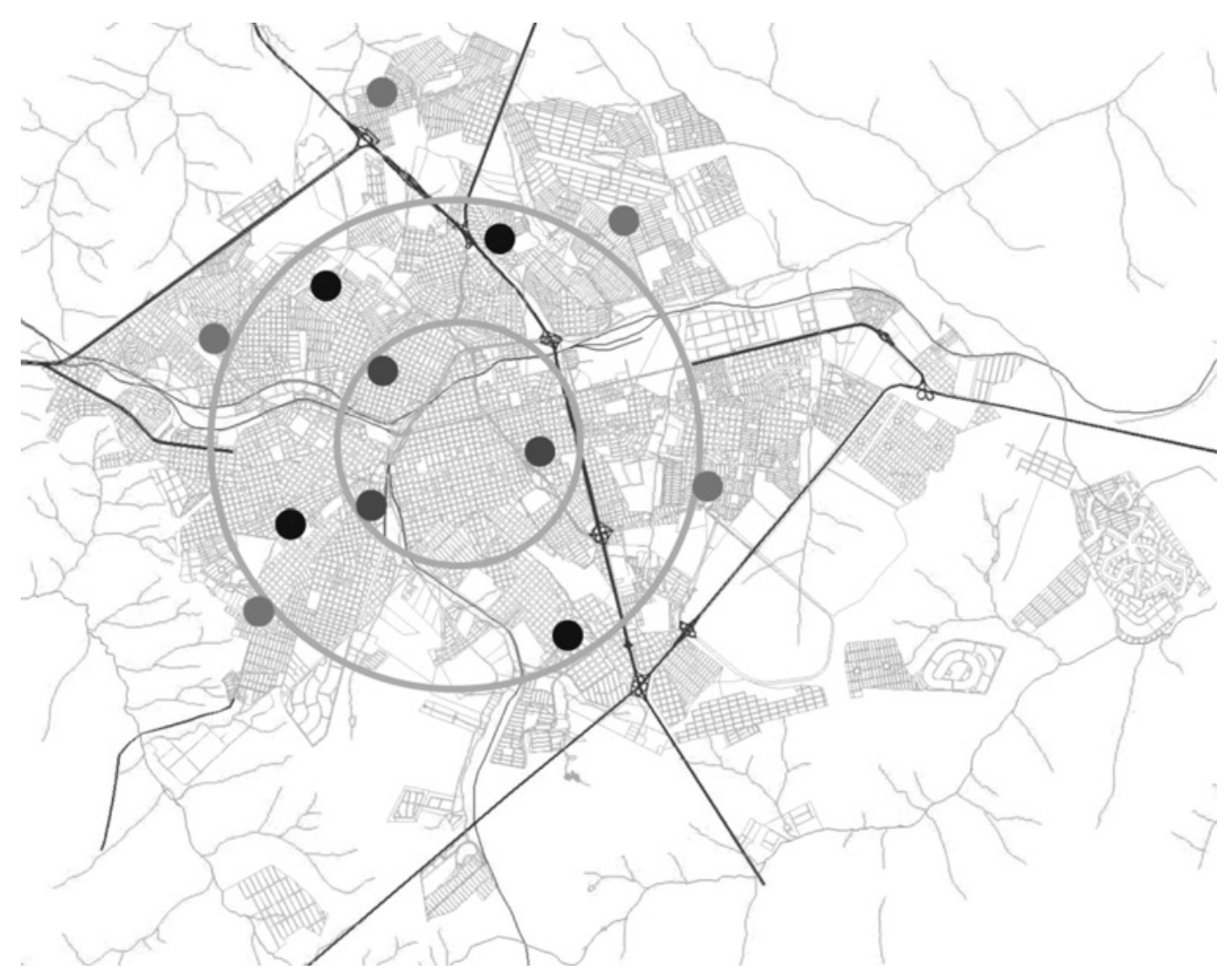

Figura 1. Mapa da cidade de Bauru, divisão dos três setores e representação das doze escolas estaduais que participaram da amostragem. 
Quadro 1. Classificação clínica do tracoma para diagnóstico epidemiológico, segundo a Organização Mundial de Saúde

\begin{tabular}{ll}
\hline Forma clínica & Descrição \\
\hline TF & Presença de 5 ou mais folículos $>0,5 \mathrm{~mm}$ na conjuntival tarsal superior \\
$\mathrm{TI}$ & Espessamento inflamatório da conjuntiva tarsal superior que obscurece mais da metade dos vasos tarsais profundos \\
$\mathrm{TS}$ & Presença de cicatriz na conjuntiva tarsal superior \\
$\mathrm{TT}$ & Presença de pelo menos um cílio tocando bulbo ocular ou indícios de epilação recente de cílio invertido \\
$\mathrm{CO}$ & Opacidade corneana que atinge a área pupilar \\
\hline
\end{tabular}

A prevalência de tracoma na população estudada foi de 3,8\%, ou seja, foram detectados 66 casos entre 1.749 crianças examinadas. Um caso foi diagnosticado como tracoma folicular/ tracoma intenso (TF/TI) e os demais, como TF.

A probabilidade da população do estudo ter tracoma, adotando-se intervalo de confiança de 95\% esteve entre 2,9\% a $4,7 \%$. A probabilidade de qualquer olho ter tracoma nesta mesma população esteve entre $2,3 \%$ a 3,5\%.

A positividade para tracoma no exame laboratorial foi de 23,1\% das amostras colhidas, comprovando-se a existência do agente etiológico na população estudada.

A chance de se encontrar uma criança com tracoma foi maior nas regiões intermediária e periférica da cidade (Tabela 1).

A avaliação da distribuição espacial dos portadores de tracoma mostrou que a maioria das crianças que estudam no centro da cidade e que foram detectadas como portadoras de tracoma, residiam em outros setores, principalmente na região intermediária e periférica (Figura 2).

Todas as crianças receberam tratamento (Azitromicina $20 \mathrm{mg} / \mathrm{kg}$ de peso em dose única) e acompanhamento como preconizado pela OMS. Os comunicantes foram examinados entre abril de 2005 e abril de 2006 e também receberam tratamento. O número de casos na cidade de Bauru para o período estudado contabilizava 261 casos.

\section{DISCUSSÃO}

A escolha da cidade de Bauru para a pesquisa se deu pelo fato dos registros de tracoma serem nulos para este município desde 2000. A doença muitas vezes é assintomática. Classicamente, o início da manifestação clínica é uma conjuntivite folicular que pode evoluir para cicatrização conjuntival, entrópio, triquíase, afinamento corneano e ulceração. Alguns pacientes desenvolvem cicatrizes corneanas que podem levar à baixa visão. O diagnóstico e o tratamento precoce na criança podem evitar a cegueira.

O trabalho de campo foi realizado no período de dois meses. Este fato é importante, tendo em vista que a população pode migrar e não se conseguir ter todos os participantes examinados caso o tempo entre o início e término da pesquisa seja grande.

As pesquisas que envolvem o tracoma em sua forma inflamatória podem ser realizadas em crianças, tanto em fase préescolar, quanto em escolares, o que motivou a realização da pesquisa nas escolas. Esta abordagem da população infantil é facilitada pelo fato das crianças frequentarem estabelecimentos de ensino, uma vez que o acesso a elas seria muito mais difícil se fossem realizados inquéritos epidemiológicos na comunidade. Desta forma, as escolas funcionam como aglomerados e este é o sistema de investigação preferido para os estudos de prevalência do tracoma inflamatório.

Os escolares em Bauru frequentavam estabelecimentos estaduais, municipais e particulares. A escolha das escolas estaduais foi feita, por se ter nestes estabelecimentos o maior número de alunos, o que daria uma abrangência maior para a
Tabela 1. Distribuição dos portadores de tracoma segundo setor de amostragem. Bauru, 2005

\begin{tabular}{lccc}
\hline & \multicolumn{3}{c}{ Tracoma } \\
\cline { 2 - 4 } Setor & Ausente & Presente & Total \\
\hline Centro & $542(98,2 \%) \mathrm{bB}$ & $10(1,8 \%) \mathrm{aA}$ & 552 \\
Intermediário & $512(94,5 \%) \mathrm{aB}$ & $30(5,5 \%) \mathrm{bA}$ & 542 \\
Periferia & $629(96,0 \%) \mathrm{aB}$ & $26(4,0 \%) \mathrm{bA}$ & 655 \\
\hline
\end{tabular}

pesquisa. Outro fator que nos fez optar por estas escolas e por não realizar a pesquisa nas escolas particulares foi que nestas últimas as pesquisas são mais dificilmente realizadas, muitas vezes por logística de abordagem que é mais difícil nesta população.

A amostra, portanto, foi baseada na distribuiçãao das escolas estaduais, a que detém a maior parte dos alunos da cidade e possui estabelecimentos nas três regiões do estudo, não havendo prejuízo na intenção do planejamento.

A prevalência de tracoma foi de 3,8\%, que segundo a OMS, coloca Bauru entre as regiões com baixa prevalência da doença ${ }^{(8)}$. Assim como Botucatu, cidade próxima, onde a prevalência no ano de 2005 entre escolares foi de 2,9\%(9).

O diagnóstico clínico é o mais importante para a constatação do tracoma, sendo considerado o melhor critério para o diagnóstico de $\operatorname{casos}^{(10)}$. Entretanto, para confirmação da existência de tracoma em locais onde não haviam casos anteriormente relatados, a Secretaria de Saúde do Estado de São Paulo preconiza a realização de exames laboratoriais, o que foi feito usando o método da imunofluorescência direta (IFD) ${ }^{(10)}$.

Assim, foram colhidas, durante a investigação epidemiológica, lâminas de raspado conjuntival de indivíduos com diagnóstico clínico de TF/TI realizando-se IFD, exame que é considerado o mais adequado para a identificação do agente etiológico, empregado para confirmação da circulação deste agente em uma comunidade e para o estabelecimento da existência de focos do tracoma ${ }^{(11)}$.

Portanto, os 23,07\% de positividade no exame laboratorial foram suficientes para comprovar a existência do agente na população estudada. Outro estudo mostrou taxa muito menor de positividade no exame laboratorial $(8 \%)^{(12)}$, apesar de taxas maiores também serem possíveis $(70 \%)^{(12)}$

É conhecido que o tracoma afeta mais pessoas de baixo nível socioeconômico e que vivem em condições de pobre$z^{(13)}$. Desta forma, a maioria dos relatos da doença aponta para "bolsões" de tracoma, localizados, em geral, nas regiões periféricas da cidade ou da zona rural onde existem mais pessoas carentes ${ }^{(14-15)}$. Este tipo de distribuição da doença que apresenta particularidades do meio ambiente e da condição das pessoas que nele habitam, fez com que tivéssemos a preocupação de definir regiões da cidade para a realização da pesquisa.

Com relação a este ponto, foi muito interessante ter sido realizado um estudo prévio também de prevalência, na cida- 


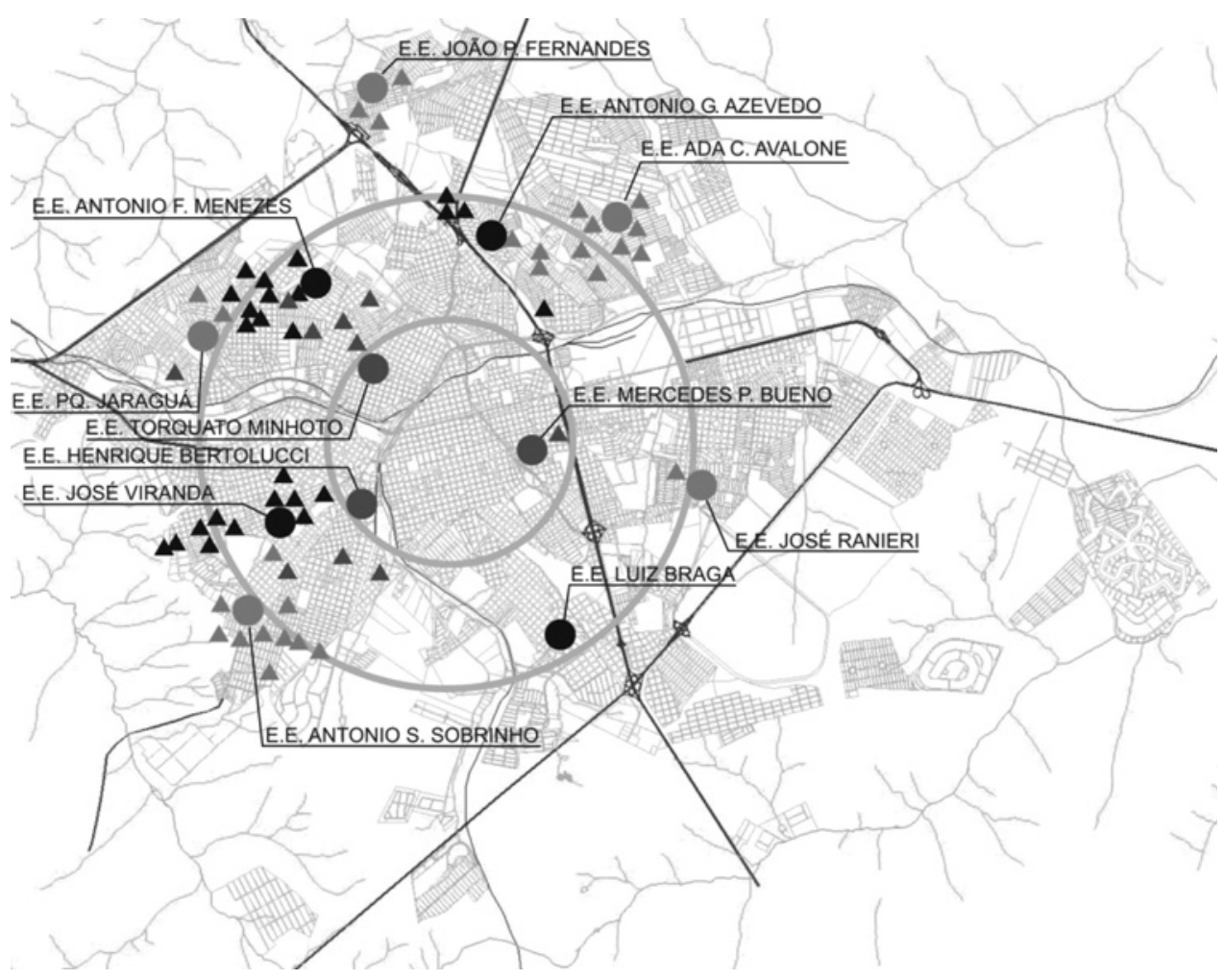

Figura 2. Distribuição dos casos de tracoma (triângulo) e escolas sorteadas (círculos) segundo setores: periferia (cinza claro), intermediária (preto) e centro (cinza escuro).

de de Bauru, e que definiu a existência de três setores distintos no Município, diferindo entre si pela condição socioeconômica e saneamento básico ${ }^{(16)}$. Os setores que foram utilizados no presente estudo seguiram exatamente a definição feita no estudo de Lombardi: região central, intermediária e periferia(16). Assim, a amostra do presente estudo foi estratificada nos setores para avaliar a possibilidade de distribuição preferencial da doença nas diferentes regiões da cidade, demonstrando-se que o tracoma foi mais frequente nas regiões intermediária e periférica da cidade de Bauru. Casos residuais e fontes de infecção podem persistir nas camadas mais pobres da população, que apresentam hábitos de higiene inadequados, e entre familiares de migrantes de áreas endêmicas que mantenham vínculo social com suas comunidades de origem $^{(17)}$.

Outro ponto importante foi o geoprocessamento que apontou o local de moradia dos alunos detectados. Muitas vezes a moradia não é próxima da escola, como pudemos constatar.

A detecção dos contatantes, assim como o tratamento dos mesmos faz parte da normativa da OMS para o tracoma. O tratamento e seguimento dos casos positivos devem ser feitos até a alta clínica, atitude que sempre deve ser tomada quando se cuida desta afecção.

Para a realização de pesquisas semelhantes a esta que está sendo apresentada é muito importante ter a colaboração da comunidade e de ter os membros das escolas trabalhando em conjunto com a equipe executora. Também é importante sincronizar as ações com a Diretoria Regional de Saúde e Secretaria de Estado de Saúde do Estado de São Paulo, que dá suporte, orienta e fornece material (manual e folheto explicativo). Este é o tipo de atitude necessária para que os projetos investigativos possam ser realizados a contento e que a população possa realmente receber os benefícios por ter participado da investigação.

\section{CONCLUSÃO}

A prevalência de tracoma em escolares de primeira à quarta série em escolas estaduais, na cidade de Bauru, São Paulo, é de 3,8\%. O estudo possibilitou que os profissionais de saúde passassem por treinamento para detecção e tratamento do tracoma, tendo sido elaborado um programa de controle de tracoma em Bauru - São Paulo. Desta forma, a pesquisa estimulou medidas de prevenção da cegueira em Saúde Ocular.

\section{AGRADECIMENTOS}

A Secretaria de Saúde do Município, ao Departamento de Água e Esgoto, a Diretoria Regional de Ensino de Bauru pelo apoio no planejamento e execução da pesquisa.

\section{REFERÊNCIAS}

1. Collier LH. Trachoma and chlamydial diseases. In: Cox FEG, editor The wellcome trust illustraded history of tropical diseases. London: The Wellcome Trust; 1996.v.1, p.83-95.

2. Jones BR. The prevention of blindness from trachoma. Trans Ophthal Soc UK. 1975 95(1):16-32.

3. Luna EJA, Medina NH, Oliveira MB. Vigilância epidemiológica do tracoma no Estado de Säo Paulo. Arq Bras Oftalmol. 1987;50(2):70-9.

4. Toledo AS. Estatística e distribuição do tracoma no Estado de São Paulo. Arq Bras Oftalmol. 1939;2:53-64.

5. Luna EJA. Epidemiologia do tracoma no Estado de São Paulo [dissertação]. Campinas: Faculdade de Ciências Médicas, Universidade Estadual de Campinas; 1993.

6. Goodman LA. Simultaneos confidence intervals for contrast among multinomial populations. Ann Math Stat. 1964;35(2):716-25.

7. Goodman, LA. On simultaneous confidence intervals for multinomial proportions. Technometrics. 1965;7(2):247-54 
8. Solomon AW, Zondervan M, Kuper H, Buchan JC, Mabey DC, Foster A. Trachoma control: a guide for programme managers. Geneva: World Health Organization; 2005.

9. Schellini SA, Lavezzo MM, Ferraz LB, Olrich Neto J, Medina NH, Padovani CR. Prevalência e localização espacial dos casos de tracoma detectados em escolares de Botucatu, São Paulo - Brasil. Arq Bras Oftalmol [Internet]. 2010 [citado 2010 Out 10];73(4):358-62. Disponível em: http://www.scielo.br/pdf/abo/v73n4/v73n4a12.pdf

10. Medina NH, Gentil RM, Caraça M, Suzuki CK, Melles HH. Análise de exames de imunofluorescência direta para o diagnóstico de tracoma. Rev Saúde Pública. 1996; 30(2):135-40.

11. Barros OM, Luna EA, Medina NH, Gentil RM. Manual de controle do tracoma. Brasília: Ministério da Saúde, Fundação Nacional da Saúde; 2001

12. Thein J, Zhao P, Liu H, Xu J, Jha H, Miao Y, et al. Does clinical diagnosis indicate ocular chlamydial infection in areas with a low prevalence of trachoma? Ophthalmic Epidemiol. 2002;9(4):263-9.
13. Caligaris LS, Morimoto WT Medina NH, Waldman EA. Trachoma prevalence and risk factors among preschool children in a central area of the city of São Paulo, Brazil. Ophthalmic Epidemiol. 2006;13(6):365-70

14. Cruz AA, Medina NH, Ibrahim MM, Souza RM, Gomes UA, Goncalves GF. Prevalence of trachoma in a population of the upper Rio Negro basin and risk factors for active disease. Ophthalmic Epidemiol. 2008;15(4):272-8.

15. Alves AP, Medina NH, Cruz AA. Trachoma and ethnic diversity in the upper Rio Negro Basin of Amazonas State, Brazil. Ophthalmic Epidemiol. 2002;9(1):29-34.

16. Lombardi HF. Oportunidades de diagnóstico precoce do HIV em gestantes do município de Bauru [dissertação]. Botucatu: Faculdade de Medicina, Universidade Estadual Paulista; 2002.

17. D'Amaral RKK, Cardoso MRA, Medina NH, Cunha ICKO, Waldman EA. Fatores associados ao tracoma em área hipoendêmica da Região Sudeste, Brasil. Cad Saúde Pública [Internet]. 2005 [citado 2008 Out 12];21(6):1701-8. Disponível em: http:// www.scielosp.org/pdf/csp/v21n6/07.pdf

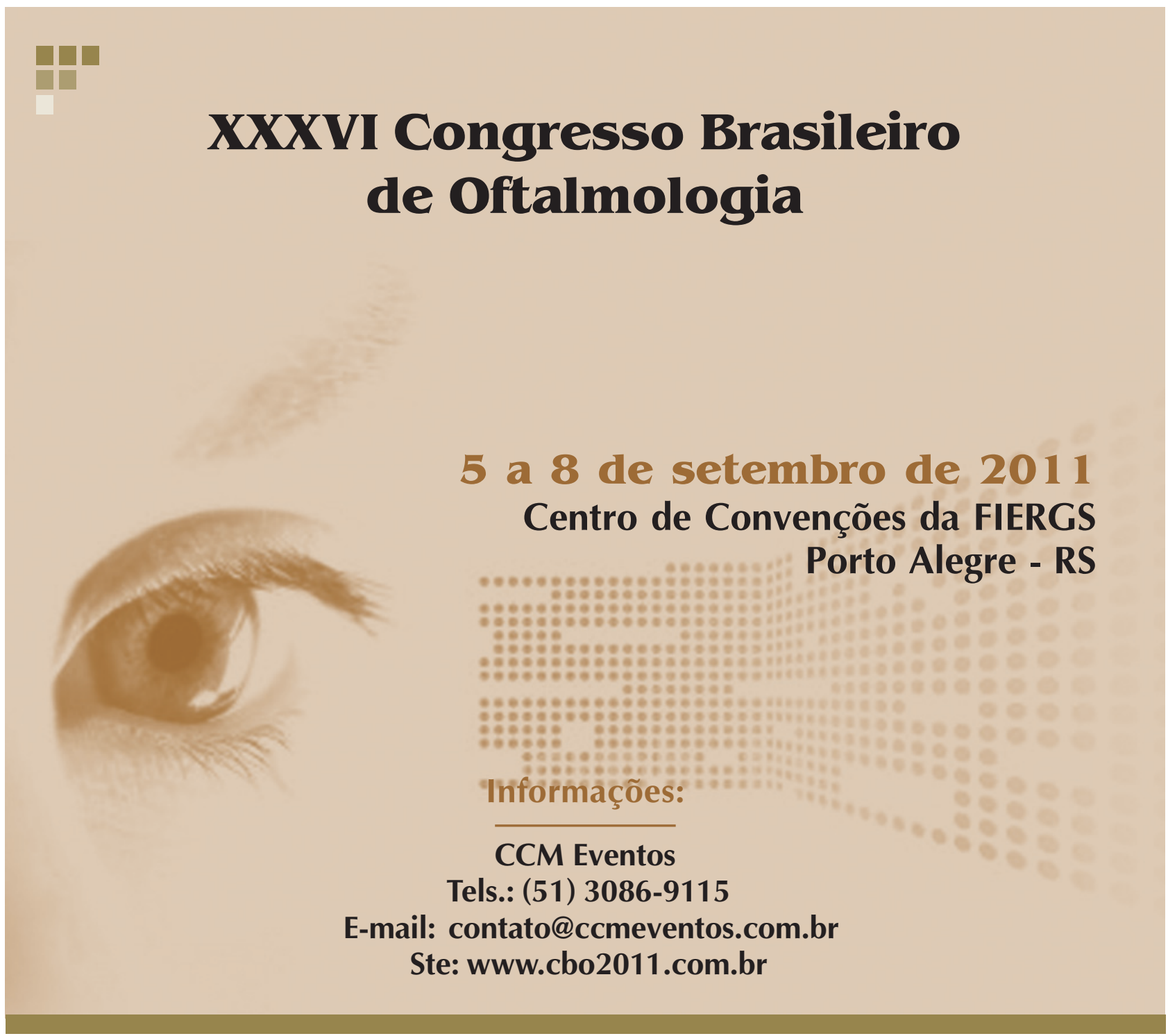

